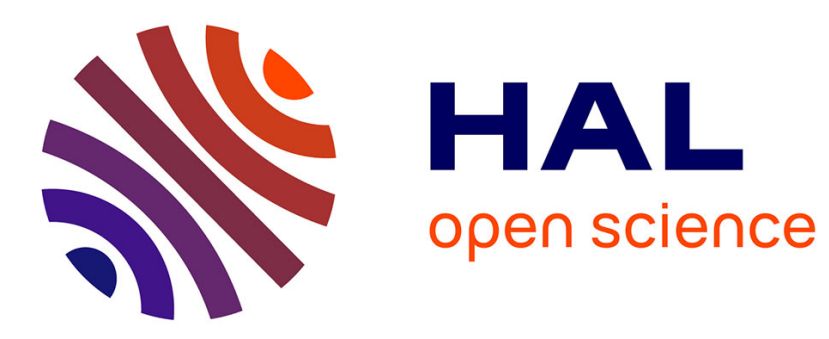

\title{
Some Introductory and Historical Remarks on Mechanics of Microstructured Materials
}

Francesco Dell'Isola, Victor A. Eremeyev

\section{To cite this version:}

Francesco Dell'Isola, Victor A. Eremeyev. Some Introductory and Historical Remarks on Mechanics of Microstructured Materials. Advances in Mechanics of Microstructured Media and Structures, pp.1-10, 2018. hal-01825568

\author{
HAL Id: hal-01825568 \\ https://hal.science/hal-01825568
}

Submitted on 28 Jun 2018

HAL is a multi-disciplinary open access archive for the deposit and dissemination of scientific research documents, whether they are published or not. The documents may come from teaching and research institutions in France or abroad, or from public or private research centers.
L'archive ouverte pluridisciplinaire $\mathbf{H A L}$, est destinée au dépôt et à la diffusion de documents scientifiques de niveau recherche, publiés ou non, émanant des établissements d'enseignement et de recherche français ou étrangers, des laboratoires publics ou privés. 


\title{
Some Introductory and Historical Remarks on Mechanics of Microstructured Materials
}

\author{
Francesco dell'Isola and Victor A. Eremeyev
}

\begin{abstract}
Here we present few remarks on the development of the models of microstuctured media and the generalized continua.
\end{abstract}

\section{Structured Media i.e. Modeling Complexity: A Change of Paradigm?}

In recent literature a great attention has been paid to the so called "structured media". These are media in which the macroscopic behavior is dictated by the micro structure of the considered systems. Of course the related theory of structures, which was originated for describing the behavior of bridges and building, then applied to airplanes, spaceships and robotics uses methods and techniques which are very close. Therefore the most modern theory did exploit the results and the ideas developed in the elder one.

The standard Cauchy continuum theory is clearly not suitable to describe the physical behavior of structured continua. For a detailed discussion of this point the reader can refer for instance to [1-4].

For this reason different generalizations were proposed. The works which we consider more enlightening and systematic are those due to Germain and Eringen (see [5-7]) and we refer to [1, 4, 8-16] and references there cited for a more detailed discussion of the explored theoretical possibilities.

The ideas of Cauchy having found brave champions (see e.g. [17]) a part of the community of mechanicians did believe that the whole continuum mechanics was

\footnotetext{
F. dell'Isola

Università di Roma "La Sapienza" and International Research Center on Mathematics and Mechanics of Complex System (M\&MOCS), Università dell'Aquila, L'Aquila, Italy e-mail: francesco.dellisola@uniroma1.it

V. A.Eremeyev (承

Faculty of Civil and Environmental Engineering, Gdańsk University of Technology, ul. Gabriela Narutowicza 11/12, 80-233 Gdańsk, Poland

e-mail: eremeyev.victor@gmail.com
} 
covered by what is indeed a particular case. Cauchy first gradient continua, while representing a particular and very important case of continua, do not exhaust all logical and physical possibilities, when one decides to introduce a continuous model for deformable bodies (see e.g. [4, 5, 7, 18]).

This circumstance had as a consequence that mechanics needed to experience (in a small scale and in a limited subgroup rather localized in space and time) a rather sharp change of paradigm (see for the discussion of the concept of paradigm and for the particular paradigm change considered here e.g. [8, 19-25]) in order to recover the capacity of describing complexity via continuum models.

The complexity at micro level do require extra kinematic descriptors at macro level: sometimes it is enough to introduce as deformation measures higher gradients of displacements (see e.g. $[6,18]$ ) but in general other completely independent descriptors are needed. If one limits to continua where it is enough to introduce balance of torque then one gets Cosserat continua (see the founding work [26] and e.g. the elegant contribution by [24, 27-32]).

Otherwise one needs variational principles and a family of new and completely independent kinematic descriptors as discussed e.g. in [5, 7, 18, 33, 34].

A possible way for getting well posed theory may consist in introducing homogenization techniques, by starting from a discrete or continuous micro-model being characterized by simple mechanical properties and complex geometry.

Gabrio Piola (translated in [35]) seems to have been one of the first scholars in proposing a kind of asymptotic homogenization and actually he ended by introducing peridynamics and high gradient models (see e.g. [1, 4, 8, 19]).

More recently more rigorous homogenization procedures are applied and used (see e.g. the fundamental book [36-49]) based on various functional analytical concepts, including Gamma-convergence. All these procedures however, while gaining in mathematical rigor, do not supply in general a heuristic tool: they are only to be used in order to justify mathematically a conjecture which has to be obtained via some other conceptual tools. Moreover the great majority of them assume some starting hypotheses which lead and limit to first gradient continua. However it has been proven that higher gradient models can be obtained via homogenization of highly contrasted micro structures (see e.g. [3, 50-61]). The conjecture which leads to these class of continua by means of asymptotic expansions is simple: different elasticity coefficients must be rescaled with the expansion parameter to diverge with suitably large power. This statement formulates precisely the condition of "presence of high contrast" in material parameters. This situation is exactly as for layered plates and shells with hight contrast in elastic moduli of layers. For low contrast the classic model of shear-deformable plates and shells can be applied whereas for high contrast the extended models that is layer-wised ones are necessary, see [62, 63].

It is worth to mentioned that the homogenization is a reach source for constitutive models of extended continue. For example it was used for derivation of the constitutive relations of micropolar solids in [33, 64-74].

The homogenization techniques can be applied also in in the case of microstructured coatings such as applied to produce superhydro- and oleo-phobic surfaces in order to obtain the models of surface elasticity developed by $[75,76]$ and by $[77$, 78], see e.g. [79]. 
It has to be remarked that in statistical mechanics some rigorous results are obtained which prove rigorously how one can get fluid continua from discrete microscopic systems (see the exhaustive reviews presented in [80-82]).

Actually while one can obtain relevant and rigorous results in homogenizing microscopically inhomogeneous systems, also including in the picture thermal phenomena for systems behaving macroscopically as fluids (see the e.g. [83-87]) and also for different class of biological systems (see e.g. [88, 89]), it is an open problem the determination, via statistical mechanics and rigorous reasonings, of micro properties leading, at macro level, to solid behavior.

Therefore one has to expect that heuristic methods, mixed micro-macro approaches and ad hoc phenomenological continuum models still play a relevant role in the efforts for describing structured media.

\section{Did Piola Formulate Already A Method of Asymptotic Homogenization?}

While Piola did not produce results comparable in rigor to the results cited in the previous introduction (clearly the concept of rigor in mathematical proof is changing in time and in space) he did formulate generalized continuum model, like those presented in this volume.

Homogenization procedures start by considering two different levels at which modeling is performed. We talk about respectively micro and macro descriptions and they correspond to relatively smaller or larger length scales.

As we have discussed there are two different class of homogenization results: the first class of these results is based on heuristic considerations leading to conjecture, having accepted more or less clear assumptions, that a specific set of discrete micro models, if the characteristic length-scale tends to zero, can converge to a certain macro continuous model, at least under well specified phenomenological conditions. If one is lucky (or clever enough) these heuristic analyses can lead to the identification of continuum constitutive parameters in function of the properties of the micro discrete systems.

The second class consists in producing precise mathematical results: the micro problem and the limit macro problem are compared and the difference of their solutions is estimated by introducing an upper bound, which, eventually, is proven to vanish when the homogenization parameter tends to zero. As the methods used in this second class are modern they did not belong to the generation of mathematicians of Piola: it is useful to distinguish them as results based either on the functional analysis [36, 37, 45, 49], or on Gamma-convergence (see e.g. [3, 39, 41, 42, 50, 51, $53,54]$ ), or on a priori estimated techniques (see e.g. [55]).

Remark that when one starts from a micro model one can reach a macro model only after having accepted suitable kinematical and simplifying assumptions. These assumptions actually characterize the macro target model and this macro model is 
assumed to be able to describe the overall behavior of the micro model only in some and well specified physical instances.

Actually there are (eventually infinite) many micro motions which can correspond to a unique specifically chosen macro motion: in statistical mechanics and in any homogenization procedure one has to make a limiting choice of one specific class of micro motions for each macro motion in order to develop the macro model: this is an intrinsic characteristic of the "averaging" procedure.

Based on this correspondence one must also try to identify the evolution equations of the macro motion starting from the geometrical and mechanical properties of micro system.

We stress that it has to be recognized that Piola's contribution to mechanics must include his identification of a micro motion for a discrete lattice material particles systems in terms of a macro motion as given by the placement fields for the homogenized continuum, as functions of space and time.

This is exactly the method which he uses to formulate (see [4]) his generalized continuum macro models, which are very advanced even nowadays and which has been rediscovered more than 150 years later.

Of course one can object that Piola did not "prove" that starting from micromotions, as solutions of micro evolution equations, and considering macro-motions, as solution of macro evolution equations, when the chosen micro-level length-scale tends to zero, the micro motions tend to produce exactly the considered macro motions, once a suitable way for measuring their distance has been introduced.

This procedure requires to preliminary find a well-posedness result for both micro and macro problems and the capacity of finding a functional space to which both macro and micro motions belong. Many different techniques have been developed: see e.g. the following references: ([3, 36, 37, 39, 41, 42, 45, 49-51, 53-55]). The reader will not be surprised to see that considered models at micro scale are sometimes infinite dimensional continuous and other times finite dimensional models: macro models, instead, are nearly always continuous.

Using his identification procedure Piola manage to formulate continuum models which are more general than those studied by Cauchy: however these last class of models attracted the attention of the majority of engineering scientists and therefore when both at micro and macro levels one considers Cauchy (i.e. first gradient) continua it is possible to find, in modern literature, many interesting results which frame homogenization in very advanced mathematic al theories: Gamma convergence and many other functional analysis concepts were developed under the push of the demands from this mechanical problem: the reader is referred for more details, for instance, to [36, 37, 39-47, 49, 90]. All mathematical results in this context are based on the rigorous proof of the convergence of micro energies to macro energies when the introduced micro scale vanishes.

It has to be remarked that Piola considers at micro-level a system of particles placed in a lattice: they may experience also long range interactions and their placement may be changed under the action of external actions. As long range interactions among particles are allowed the continuum limit which he obtains results to be a generalized continuum. Remark that Piola did not know that subsequent literature would 
be bound to consider Cauchy particular class of continua: therefore he calls what in modern times are "generalized continua" simply "continua". Piola's continua, actually were bound to be rediscovered in XXI century: they are the object of the study in so called Peridynamics (see for a more detailed discussion the work [4]).

Piola seems to attribute to discrete systems a specific and relevant physical meaning, as was customary in his époque. Later science was shacked by the terrible controversy between Mach and Boltzmann. It seems to us that Piola would have shared the ideas expressed by Boltzmann. It has to be remarked that Piola had no ideas of the true dimensions of atoms and molecules and that his considerations do not include temperature. Some homogenization results (and very rigorous ones) have been obtained, for discrete systems, in statistical mechanics. With these results one can prove rigorously how fluid continua can be seen as a limit of discrete microscopic systems, see the interesting reviews in [80-82]. These results in homogenizing microscopically inhomogeneous systems, which manage to interpret thermal phenomena in systems which behave macroscopically as fluids (see e.g. the papers [83-87]) and in some biological systems (see e.g. [88, 89]), were not extended, up to now, to determine, by means of rigorous statistical mechanical methods, which are those micro properties which produce, at macro level, deformable solids.

As a consequence the old fashioned heuristic methods à la Piola can still be topical, as it may represent the only available conceptual tool to model some specific complex mechanical systems. An example of their application is presented in [9193], while in [58, 59, 91] some mixed micro-macro approaches are attempted. Also numerical identifications (see e.g. [94, 95]) and ad hoc phenomenological continuum models in $[2,5,26,58,59,91-93,96,97]$ still play a relevant role in the efforts for describing structured media.

Exactly as happens in statistical mechanics the system that Piola considers at micro level is finite dimensional: in modern literature one finds less attention to the homogenization of this kind of models (see e.g. [38, 39]).

We believe that starting from lattices of interacting particles it is more natural to arrive to macro homogenized models which do not verify the basic assumptions by Cauchy: and indeed Piola did easily formulate such a kind of models. Also it has to be noticed that the modern debates and discussions questioning the use and the role of second or higher gradient continuum theories in mechanical sciences are not new.

Francesco Brioschi edited the last work by Piola translated in [35], posthumous in 1856: in the title of this work the word "controversial" appeared in a evident position. Cauchy, Navier and Poisson, changing the direction given to the French mechanics school by Lagrange and D'Alembert, did not believe any more to the powerful heuristic importance of variational methods.

More recently the French school (leaded by Paul Germain see e.g. [6, 7]) came back to the ancient ideas by Lagrange, as divulged by Piola.

Therefore we have an apparently paradoxical situation: even if the results presented by Piola are not as rigorous as required by the modern standards in the mathematical theory of homogenization, they are often, and by far, more general than those presented in the great majority of recent papers and textbooks in continuum mechanics. 
We believe that the Piola's micro-macro identification procedure can be easily used as a heuristic guidance to new and very interesting rigorous results: Piola identifies macro virtual work in terms of macro kinematical descriptors by imposing its equality with micro virtual work. To do so he assumes what we want to call Piola's Ansatz:

(i) one assumes that a smooth macro displacement field exists which describes at this length scale the overall behavior of studied complex system,

(ii) one identifies the displacements of the particles forming the micro-lattice by means of the values of the macro displacement in the nodes of the lattice;

(iii) he identifies finite differences with derivatives and in this way he identifies the expression of macro deformation and kinetic energy and macro virtual work in terms of micro geometrical and physical properties.

In our opinion this process is due to Piola (again we are ready to discover a further occurrence of Stigler's law) and we call it: Piola identification process.

\section{Variational Principles as a Guide for Formulating New Models: The Case of Hencky and Euler Beams}

Which postulation scheme is needed when one needs to formulate new models for describing the physical behavior of systems not yet studied?

Many possible epistemological choices can be used: D'Alembert, Lagrange and Piola are among the founders of a school whose postulation is based on variational principle. The most general of such principles is the Principle of Virtual Work, of which the Hamilton Principle can be regarded as a particular case.

It is relevant to understand when this principles were introduced at first. In our opinion in many cases new mathematical models could be formulated only after having approached the problem by using the postulation scheme based on variational principles. For historical remarks on various variational principles we refer also to [98].

This opinion can be tested only by checking all possible instance of novel models, as introduced in different époques: of course in this context it is essential to determine the first formulation of a given model. Indeed once formulated a models, for instance starting from a variational principle, it is easy to reformulate it by assuming a postulation scheme based on balance principles.

From an epistemological point of view it is essential to establish if the discovery is more frequent when one starts from variational principles or if he starts from balance principles.

Therefore while the fight for priority may be futile, to establish which postulation scheme was more successful may be of relevance.

We discuss here two examples. 


\subsection{First Formulations of Hamilton Principle}

For sure in the work by Piola published in 1825 one finds the following statement:

"Concluderemo adunque che le funzioni di $t$ volute dalle leggi della natura e che esprimono le coordinate di tutti i punti del sistema alla fine di un tempo qualunque, hanno quelle stesse forme che rendono massima o minima la primitiva della funzione $\Omega-U-\frac{1}{2} \sum\left(d x^{2}+d y^{2}+d z^{2}\right)$ definita fra i due limiti valori del tempo".

This statement can be translated as follows:

"We will therefore conclude that the functions of the time $t$ which are wanted by the laws of nature and which express the coordinates of all the points of the system at the end of any time whatsoever, have exactly the same forms which render maximal or minimal the primitive of the function $\Omega-U-\frac{1}{2} \sum\left(d x^{2}+d y^{2}+d z^{2}\right)$ as defined between the two limit values of the time".

Few years later such a statement has been called "the Principle of Hamilton". The reader will remark that this variational principle has been placed at the basis of the mechanics of material bodies in the two papers "On a General Method in Dynamics" which were published in the Philosophical Transactions in 1834 and 1835 (see [99]).

In [99] Hamilton writes: "But when this well known law of least, or as it might be better called, of stationary action, is applied to the determination of the actual motion of the system, it serves only to form, by the rules of the calculus of variations, the differential equations of motion of the second order, which can always be otherwise found." ....

Therefore it seems that also Hamilton needed to discuss and support his variational point of view, if he feels the need to support it by confirming its results with those obtained in another way.

"A different estimate, perhaps, will be formed of that other principle which has been introduced in the present paper, under the name of the law of varying action, in which we pass from an actual motion to another motion dynamically possible, by varying the extreme positions of the system, and (in general) the quantity $\mathrm{H}$, and which serves to express, by means of a single function, not the mere differential equations of motion, but their intermediate and their final integrals."

In reading Hamilton one must deduce that he did not know Piola's previous work. There are no reasons to doubt about this fact: indeed he expresses his total admiration of the work of his predecessors and in particular those due to Lagrange. He claims:

\footnotetext{
The theoretical development of the laws of motion of bodies is a problem of such interest and importance, that it has engaged the attention of all the most eminent mathematicians, since the invention of dynamics as a mathematical science by Galileo, and especially since the wonderful extension which was given to that science by Newton. Among the successors of those illustrious men, Lagrange has perhaps done more than any other analyst, to give extent and harmony to such deductive researches, by showing that the most varied consequences respecting the motions of systems of bodies may be derived from one radical formula; the beauty of the method so suiting the dignity of the results, as to make of his great work a kind of scientific poem.
}

The previous excerption by Hamilton expresses an opinion fully shared by Piola. Both Hamilton and Piola are to be considered immediate successors of Lagrange in 
the leadership of the mechanical school basing the study of physical systems on a postulation which has as central point the Principle of Hamilton, or its weaker form (later used systematically by Piola) given by the Principle of Virtual Work.

We can easily accept that Hamilton and Piola are among the brightest scientists in mechanical sciences. They both regarded themselves as continuators of the work of D'Alembert, in the spirit (i.e. following his metaphysics, using Piola's expression) of Lagrange.

We cannot be sure that Piola is actually the true originator of modern generalized continuum theories: we are aware of the fact that in [100], Stigler's law of eponymy is formulated "no scientific discovery is named after its original discoverer".

\subsection{Hencky Discrete Models and Euler Continuum Models for Beams}

In 1825 Piola published his first scientific Memoir. Piola considers more fundamental and more physically understandable a model for planar beams based on the consideration of an "equivalent" system of bars and concentrated springs. He regards an Euler beam as the limit of a set of bars: each bar can be elongated and the relative angle between two bars can change. Both elongation and angle variations determine a variation of deformation energy, whose expression in left arbitrary. Actually Piola formulates a principle of virtual work by specifying suitable deformation energies and by determining the dual quantities of these deformations.

Piola's discrete model will be studied by Hencky nearly one century later, regarding it as an approximation of Euler beam. Piola regards as more understandable the described discrete system: he finds possible the formulation of the Euler model only regarding it as a limit of a discrete system of bars. Both Piola and Euler (see e.g. [101] for a detailed discussion of the original works of Euler and his choice of basing his investigations on variational principles) systematically formulate, as the most fundamental assumption, a principle of virtual work.

Consider that Euler was among the first authors studying the deformation of Elasticas and that Piola is one of the first authors studying Hencky type models for beams.

We can read some excerptions from the Memoir of 1825 by Piola, when he talks about elastic curves:

In the elastic curves it occurs to have to consider internal forces as those recalled in the $\mathrm{n}$ 199; they can be, as we will see, of three kinds; but if one wants to conceive their action, it is possible to do so only by using the approximating polygon (193).

It is obvious then that for Piola the ontological reality resides only in discrete models, while the continua are only some mathematical tools which can be of use when looking for the solution of specific applicative problems: he relies for this on the powerful methods of mathematical analysis.

Piola continues by stating that: 
Therefore this is what we will do, by recalling that it is not important to be able to form in our mind the image of the way of action of elastic forces in the curves: for what concerns the effects, they can be found as could be found if it would not occur (occurrence which still is indispensable) the disappearance of every representation in the passage from the polygon to the curve, which is its limit (see again 193).

Here Piola states clearly the fact that he refrains from the consideration of internal forces: he considers the efforts in this direction "not important".

Finally he lists the three kinds of elasticity which can be observed in elastic curves:

At first the one related to elongations:

The first kind of elasticity, to which one wanted to give the name of tension, is that elasticity for which, even if there were not external applied forces, one would change every side of the subtended polygon by elongation or contacting it.

then the kind related to bending

The second kind of elasticity, or that kind of elasticity to which the recent writers have left the name without any specific adjective, is imagined to act on the angle formed by two consecutive sides of the polygon and along all the polygon itself. However, in order to find the term which this kind of elasticity has introduced in the general equation, it is convenient to determine, at first, the function $F$ (199) which said elasticity tends to make vary.

Piola manages to determine some measures of deformation able to account for the bending, starting from the placement function of the elastic curve. In [102] the spirit of Piola is evoked to find an expression of continuous deformation energy and to formulate the equilibrium minimization problem for pantographic structures. Exactly as forecast by Piola the obtained models are generalized second gradient continua.

Then Piola proves that the limit of his Hencky type discrete beam leads exactly to the fundamental conjecture by Euler and Bernoulli: bending deformation energy depends on the curvature of the elastica.

Again quoting Piola (his Memoir 1825):

Therefore (199) in the term $S E \delta f$ introduced by that elastic force the $f$ will have the just determined value. As then, being $R$ the radius of curvature, one knows that

$$
R=\frac{\left(D x^{2}+D y^{2}+D z^{2}\right)^{\frac{3}{2}}}{\sqrt{\left\{\left(D x D^{2} z-D z D^{2} x\right)^{2}+\left(D y D^{2} z-D z D^{2} y\right)^{2}+\left(D x D^{2} y-D y D^{2} x\right)^{2}\right\}}}
$$

in this way it can be seen how we get

$$
f=\frac{D s}{R} \quad \text { " }
$$

To complete his analysis Piola considers beams having non planar actual configurations: 
Mister Binet ( $\mathbf{J})$ was the first author who introduced the consideration of a third kind of elasticity, and he wanted to call it torsion: this kind of elasticity had escaped the analysis of Lagrange, and nevertheless one cannot deny that it can be observed also in nature. To understand what it is [such a concept] let us consider three consecutive sides of the subtended polygon (199), and it is very well clear that the plane formed by the first two sides will not generally be the same plane which contains the last two sides: these two planes will form an angle and it is on this angle that the new elasticity is intended to act. Once called $F$ this angle of the polygon, if we will manage to transform it into the usual form $\omega f+\omega^{2} k+$ etc., we will also know the term $S E \delta f$ introduced by the torsion in the general equation (199). Again here, as in the previous paragraph and for a similar reason, I will give a new way for finding this angle.

We conclude with two remarks:

(i) Piola's mathematical investigations gave an important contribution to the modern theory of one dimensional extensional continua, moving in space and capable to store bending and twisting energy;

(ii) Piola's results established the mathematical basis of the numerical study of Euler or Hencky beams, basing the formulation of their evolution equations on the firm basis of variational principles.

\section{Discrete Versus Continuous Description: An Ancient Dichotomy}

Greek philosophers debated about the true ontological nature of matter. Epicurean believed in its discrete nature, as they considered atoms as the smallest indivisible constituent of matter. On the other side Heraclitus did believe that the ontological nature of matter was intrinsically continuous.

However already Archimedes was aware of the difficulty in reaching any definite conclusion about the ultimate true nature of matter (and any other physical phenomenology): in fact he was aware that any mathematical theory had a specific use limited to describe only a well determined class of phenomena.

Therefore the question concerning discrete of continuous description has to be considered simply as a matter of opportunity: in a given context which is the most suitable approach? It seems rather difficult to establish that one description between the one give by discrete models or the other one based on continuous models is the closest to reality.

Archimedes (and the modern scientists) refrain from any effort of attaining any definite truth about the ultimate nature of matter. We must be content to find the class of model which more suitably describes a given class of phenomena. It seems that also Piola accepts such a point of view. The principle of Virtual Work is at the basis of the whole set of memoirs by Piola: all the models which he considers starts from the postulation of the internal and external work functionals. He prefers to start his analysis by considering discrete models for the lattices of particles which he wants to consider and he claims to be able to understand clearly the physical meaning of such 
discrete models: he believes that continuum models represent as mental construction useful to describe the behavior of discrete systems as a whole and only in some specific and well determined situations. Actually he considers continuous models as a mathematical tool to calculate solutions to equilibrium or motion problems: partial differential equations are considered in his époque (see also for instance the results by Hamilton leading to Hamilton-Jacobi partial differential equation) as a tool useful to calculate the solution of systems of ordinary differential equations having many unknown time varying function.

For those who may ask themselves how it is possible to believe that more complex PDEs may be of use in solving simpler ODEs we remark here that it can happen that PDEs can be solved with less efforts that the original ODEs: the French school in engineering sciences obtained wonderful results using this method, mainly when dealing with linearized equations. For instance De Saint Venant opened for engineering applications all the results for linear elasticity and had a great impact in engineering capacity of designing structures.

In our opinion, for Piola and many scientists of his generation, continuum mechanics represents a "computational tool". It is a "mathematical trick" which allows for computation of some solutions which are relevant in the engineering applications. As sometimes happens in science, what was born as a computational tool became a foundational concept: the choice of considering balance equations as the most fundamental concepts in mechanics (due probably to Cauchy, but who knows? Maybe Stigler's law applies also here) produced the consequence of considering the principle of virtual work as a less fundamental concept. This lead to the following oxymoron: "we prove now the theorem of the principle of virtual work". Further investigations are now needed to districate such an oxymoron and many other related ones.

The dichotomy discrete/continuous was present in the whole history of science and we cannot discuss in a small space it: such dichotomy has so many aspects which it could be a gigantic work simply to give a short review about its historical development. Piola surely consider atomistic models more fundamental, but we believe that he did not manage to arrive to a formulation close to modern statistical mechanics as he does not manage to conceive any kinetic concept leading to the concept of temperature and heat flux. Piola explicitly states that he develops mechanics with a postulation where Thermodynamics does not play any role. It is very important to remark that, in order to use computer aided simulations, leading to numerical prediction of considered phenomena, in general the literature one considers Euler-Lagrange equations (i.e. balance equations) as the most fundamental concepts to be postulated as basic principle. Then one multiplies by test functions (suitably regular) and after a painful integration by parts he is lead to get a variational principle for the continuous system which is considered. Finally one discretizes via a finite elements the obtained expression for internal and external work to compute, with a numerical integration scheme, the desired discrete approximation of the continuous field solution of the problem formulated with the continuous model.

This seems to be a vicious circle and therefore one may ask a question: is it really necessary to homogenize and then discretize again? Maybe it is much better to study the discrete system which we have at the beginning! It is possible, probably 
sometimes easier, to make computations based on the discrete systems which one can postulate directly without any intermediate step. This is spirit which inspired, for instance, the papers [103-108]. Indeed they central idea of the numerical simulations presented there is simple: one does not model pantographic structures with a continuum (which is necessarily to be chosen in the class of second gradient continua). Actually such continuum is inspired by a homogenization process similar to the one due to Piola and Hencky: in order to get some meaningful numerical predictions a subsequent PE discretization is needed, which must involve suitably regular elements. On the contrary a discrete Lagrangian model is postulated since the very beginning and the numerical code needed to find the motion is adapted to use directly it.

In some sense the continual models using PDEs were widely applied since these models give some very useful tool for analytical analysis whereas discrete models require almost always numerical methods. So it was one of reasons to replace a lattice model with atoms and molecules by a continual counterpart. Nowadays, there are many developments in the theory of lattices considered as discrete systems, see e.g. [109-114].

It is remarkable how some conceptual tools initially introduced to meet the needs imposed by computing (here we consider, as examples, the partial differential equations obtained as Euler Lagrange stationarity conditions or also the Ptolemaic epicycles in the calculations needed to study, in Hellenistic époque, the mechanics of celestial bodies) may have changed their role in the process of formulating theories in different cultural paradigms.

Instead of representing a tool for getting the solution of engineering problems in a given milieux they could become the most fundamental concepts in the views of some schools.

This is what happened to PDEs: being essentially equivalent to balance equations in continuum mechanics they were regarded to supply the most fundamental conceptual tool in physics. Exactly as happened to the theory of epicycles and deferents: from being a computational tool they were transformed in middle ages as the most basic concepts in the study of celestial motion (the reader can find more details in $[19,115])$.

\section{Some Conclusions: History of Mechanics as a Tool in Finding Models for Fabrics, 3D Printed Prototypes and Various Complex Systems}

Gabrio Piola has been an Italian scientist whose contributions have been generally neglected. However he has left a trace in mechanical and mathematical literature and his works were widely distributed in the main world libraries, although written in Italian (for instance in Vienna [116, 117] and at the University of Wisconsin, Google books could find many copies of his works). Hellinger was aware of some of 
his contributions (see [9-11]) and Piola was cited also in [17]. For a more detailed discussion aimed to assess the true influence of Piola on subsequent research the reader is referred to [4] and also to [8].

Piola gives a visionary presentation of variational principles and tries to teach to his successors those methods that he believes are more suitable to invent new models. He writes in "Intorno alle equazioni fondamentali del movimento di corpi qualsivogliono, considerati secondo la naturale loro forma e costituzione." page 4, as translated in [118]:

If it is well founded or not the statement that the Lagrangian methods are sufficient to the description of all mechanical phenomena, and are so powerful that they are suitable for all further possible researches, this is what shall be decided later, and before rebutting my point of view, it will be fair to leave me to expose all arguments which I have gathered to defend my point of view. I hope to clarify in the following Memoir that the only reason for which the Analytical Mechanics seemed to be insufficient in the solution of some problems, is that Lagrange, while writing the conditions for equilibrium and motion of a three dimensional body, did not detailed his model by assigning the equations relative to every material point belonging to it. If he had done this, and he could very well do it without departing from the methods imparted in his book, he would have obtained easily the same equations to which the French Geometers of our times arrived very painfully, [equations] which now are the foundation of new theories. However those results which he could not obtain, because death subtracted him to sciences before he could complete his great oeuvre, these results can be obtained by others: this is the assumption which led me to start some efforts since the years 1832 and 1835 (See the Memoir della Meccanica dei carpi naturalmente estesi inserted in the 1st Tome of Opuscoli matematici e fisici; Milano, Giusti, 1832: and the other Sulla nuova analisi per tutte le quistioni della Meccanica molecolare in the Tome XXI of these Proceedings).

Following, more or less consciously, the indication of Piola many generalized continuum or Lagrangian discrete models have been used by some successors of Lagrange:

(i) for modeling fabrics used to built reinforced composites (see e.g. [91, 93, 119123]): the complexity at micro level of such systems very often imposes the formulation of generalized continuum models at macro level; however it must be remarked that, always using Lagrangian mechanics, such complex systems may be modeled directly by means of discrete models as done for instance in [103-108]. Hencky type models, generalizing the discrete description of Euler beams, can be very useful in formulating numerical codes suitable to predict the behavior of metamaterials and complex fabrics;

(ii) to develop more sophisticated description of deformation phenomena (see e.g. $[2,18,26,48,53,56,57,59,61,97,124-127]$ and also the fundamental papers $[6,7,24])$ : this effort has been, paradoxically, blocked by the undoubted success of the simpler Cauchy continuum models whose range of applicability is, however, rather limited in the considered instances;

(iii) to guide the invention of novel meta materials by using the technology of 3D printing (see e.g. [61, 91, 93, 102, 119, 123, 126, 128]): exotic behavior forecast for generalized continua can be transformed into real phenomena 
occurring to objects whose internal architecture is so finely specified and whose microstructure is optimized for specific applications;

(iv) to obtain novel micro-macro identification results (see e.g. [3, 37, 50, 51, 53, 54, 58-60, 103-108, 121, 122, 129-131]): reduced order models are very useful to save computing time and to make possible the required predictions concerning the physical behavior of complex systems;

(v) to obtain interesting models of biomechanics phenomena (see e.g. [96, 132135]): many different length scales appear in biomechanical systems and when modeling them the standard Cauchy continuum mechanics is very often not suitable to fully describe relevant phenomena.

Gabrio Piola describes in his first published work in 1825 a homogenization procedure allowing for the determination of a continuum model for a discrete systems constituted by a lattice of particles in the reference configuration. He also obtains an identification of macro parameters when micro geometrical and mechanical properties are known. Piola's homogenization procedure has been used often in the subsequent literature as it is very useful to model the behavior of complex systems.

However we are aware of Stigler's law of eponymy: therefore we do not give as granted that Piola was indeed the first scientist who did introduce the heuristic method of asymptotic homogenization which he describes in his works.

On the other hand there are some precise statements which can be assessed:

(i) many results by Piola seem to have been rediscovered also 150 years later,

(ii) Piola variational postulation of (continuum) mechanics is more encompassing than the postulation proposed by Cauchy, between 1822 and 1850),

(iii) Piola does not use tetrahedron argument, as he bases all his analysis on the principle of virtual work,

(iv) Piola prefers to start with discrete finite dimensional particle models of mechanical complex systems, and he uses the ideas and the concepts from continuum mechanics as a purely computational tool,

(v) Piola believes that all physical theories are to be based on variational principles.

In recent times many authors discussed about the need of considering large deformation measures in engineering applications. Many claimed that only linearized theories have a "utility" in engineering applications and that finite deformations "are not needed in the engineering practice". This point of view leads to miss the physical understanding of studied systems and is contradicted by the most modern technological demands. In the works by Piola all kinematical quantities are considered for large displacements and large deformations as the are the only concepts having a true physical meaning. Many scientists needed to recover this attitude in recent times. The temporary success of linearized models has actually blocked engineering sciences in the development of very interesting applications for many years.

The papers presented in this volume share the visionary point of view of Piola in many aspects and they seem to be continuing his conceptual efforts. 


\section{References}

1. Auffray, N., dell'Isola, F., Eremeyev, V.A., Madeo, A., Rosi, G.: Analytical continuum mechanics à la Hamilton-Piola least action principle for second gradient continua and capillary fluids. Math. Mech. Solids 20(4), 375-417 (2015)

2. Bertram, A., Glüge, R.: Gradient materials with internal constraints. Math. Mech. Complex Syst. 4(1), 1-15 (2016)

3. Camar-Eddine, M., Seppecher, P.: Determination of the closure of the set of elasticity functionals. Arch. Ration. Mech. Anal. 170(3), 211-245 (2003)

4. dell'Isola, F., Andreaus, U., Placidi, L.: At the origins and in the vanguard of peridynamics, non-local and higher-gradient continuum mechanics: an underestimated and still topical contribution of gabrio piola. Math. Mech. Solids 20(8), 887-928 (2015)

5. Eringen, A.C., Kafadar, C.B.: Polar field theories. In: Eringen, A.C. (ed.) Continuum Physics. vol. IV, pp. 1-75. Academic Press, New York (1976)

6. Germain, P.: La méthode des puissances virtuelles en mécanique des milieux continus. première partie: théorie du second gradient. J. Mécanique 12, 236-274 (1973)

7. Germain, P.: The method of virtual power in continuum mechanics. part 2: microstructure. SIAM J. Appl. Math. 25(3), 556-575 (1973)

8. dell'Isola, F., Corte, A.D., Giorgio, I.: Higher-gradient continua: the legacy of Piola, Mindlin, Sedov and Toupin and some future research perspectives. Math. Mech. Solids 22(4), 852-872 (2017)

9. Eugster, S.R., dell' Isola, F.: Exegesis of Sect. II and III. A from fundamentals of the mechanics of continua by Hellinger, E. ZAMM (2017). https://doi.org/10.1002/zamm.201600293

10. Eugster, S.R., dell'Isola, F. (2017) Exegesis of Sect. III. A from fundamentals of the mechanics of continua by E. Hellinger. ZAMM

11. Eugster, S.R., dell'Isola, F.: Exegesis of the Introduction and Sect. I from fundamentals of the mechanics of continua by Hellinger, E. ZAMM 97(4), 477-506 (2017)

12. Maugin, G.A.: Generalized continuum mechanics: what do we mean by that? In: A MG, V MA (eds) Mechanics of Generalized Continua. One Hundred Years after the Cosserats, Springer, pp. 3-13 (2010)

13. Maugin, G.A.: A historical perspective of generalized continuum mechanics. In: Altenbach, H., Erofeev, V.I., Maugin, G.A. (eds.) Mechanics of Generalized Continua, pp. 3-19. From the Micromechanical Basics to Engineering Applications, Springer, Berlin (2011)

14. Maugin, G.A.: Generalized Continuum Mechanics: Various Paths, pp. 223-241. Springer, Dordrecht (2013)

15. Maugin, G.A.: Continuum mechanics through ages. From the Renaissance to the twentieth century, Springer, Cham (2016)

16. Maugin, G.A.: Non-Classical Continuum Mechanics: A Dictionary. Springer, Singapore (2017)

17. Truesdell, C., Noll, W.: The Non-linear Field Theories of Mechanics, 3rd edn. Springer, Berlin (2004)

18. dell'Isola, F., Seppecher, P., Madeo, A.: How contact interactions may depend on the shape of Cauchy cuts in Nth gradient continua: approach "à la D'Alembert". Zeitschrift für angewandte Mathematik und Physik 63(6), 1119-1141 (2012)

19. dell'Isola, F., Della Corte, A., Esposito, R., Russo, L.: Some cases of unrecognized transmission of scientific knowledge: from antiquity to gabrio piolas peridynamics and generalized continuum theories. In: Generalized continua as models for classical and advanced materials, Springer, pp 77-128 (2016)

20. Kline, M.: Mathematical Thought From Ancient to Modern Times: Voll. 1,2,3, vol. 3. OUP USA (1990)

21. Kuhn, T.S.: The Structure of Scientific Revolutions, 3rd edn. University of Chicago Press (1996)

22. Sedov, L.I.: Mathematical methods for constructing new models of continuous media. Russian Math. Surv. 20(5), 123 (1965) 
23. Stillwell, J.: Exceptional objects. Am. Math. Month. 105(9), 850-858 (1998)

24. Toupin, R.A.: Theories of elasticity with couple-stress. Arch. Ration. Mech. Anal. 17(2), 85$112(1964)$

25. Turro, N.J.: Paradigms lost and paradigms found: Examples of science extraordinary and science pathological and how to tell the difference. Angewandte Chemie International Edition 39(13), 2255-2259 (2000)

26. Cosserat, E., Cosserat, F.: Théorie des corps déformables. Herman et Fils, Paris (1909)

27. Mindlin, R.D.: Micro-structure in linear elasticity. Arch. Ration. Mech. Anal. 16(1), 51-78 (1964)

28. Mindlin, R.D.: Second gradient of strain and surface-tension in linear elasticity. Int. J. Solids Struct. 1(4), 417-438 (1965)

29. Mindlin, R.D., Eshel, N.N.: On first strain-gradient theories in linear elasticity. Int. J. Solids Struct. 4(1), 109-124 (1968)

30. Mindlin, R.D., Tiersten, H.F.: Effects of couple-stresses in linear elasticity. Arch. Ration. Mech. Anal. 11, 415-448 (1962)

31. Nowacki, W.: Theory of Asymmetric Elasticity. Pergamon-Press, Oxford (1986)

32. Toupin, R.A.: Elastic materials with couple-stresses. Arch. Ration. Mech. Anal. 11(1), 385414 (1962)

33. Eremeyev, V.A., Lebedev, L.P., Altenbach, H.: Foundations of Micropolar Mechanics. Springer, Heidelberg (2013)

34. Eringen, A.C.: Microcontinuum Field Theory. I. Foundations and Solids, Springer, New York (1999)

35. dell'Isola, F., Andreaus, U., Cazzani, A., Perego, U., Placidi, L., Ruta, G., Scerrato, D.: Di un principio controverso della meccanica analitica di lagrange e delle molteplici sue applicazioni. In: The complete works of Gabrio Piola: vol. 1 I, Springer, pp 371-590 (2014)

36. Bensoussan, A., Lions, J.L., Papanicolaou, G.: Asymptotic analysis for periodic structures, vol. 5. North-Holland, Amsterdam (1978)

37. Bakhvalov, N.S., Panasenko, G.: Homogenisation: Averaging Processes in Periodic Media: Mathematical Problems in the Mechanics of Composite Materials, Mathematical Problems in the Mechanics of Composite Materials, vol. 36. Kluwer, Dordrecht (1989)

38. Braides, A.: Gamma-convergence for Beginners, vol. 22. Clarendon Press (2002)

39. Braides, A.: A handbook of $\gamma$-convergence. North-Holland, Handbook of Differential Equations: Stationary Partial Differential Equations 3, 101-213 (2006)

40. Braides, A., Defranceschi, A.: Homogenization of Multiple Integrals, vol. 12. Oxford University Press, Oxford (1998)

41. Braides, A., Truskinovsky, L.: Asymptotic expansions by $\gamma$-convergence. Continuum Mech. Thermodynam. 20(1), 21-62 (2008)

42. Dal Maso, G.: An introduction to $\Gamma$-convergence, Progress in Nonlinear Differential Equations and Their Applications, vol. 8. Birkhauser, Basel (1993)

43. Hornung, U. (ed.): Homogenization and porous media, Interdisciplinary Applied Mathematics, vol. 6. Springer, New York (2012)

44. Jikov, V.V., Kozlov, S.M., Oleinik, O.A.: Homogenization of Differential Operators and Integral Functionals. Springer, Berlin (1994)

45. Kozlov, S.M.: Averaging of random operators. Matematicheskii. Sbornik 151(2), 188-202 (1979)

46. Målqvist, A., Peterseim, D.: Localization of elliptic multiscale problems. Math. Comput. 83(290), 2583-2603 (2014)

47. Oleinik, O.A., Shamaev, A.S., Yosifian, G.A.: Mathematical problems in elasticity and homogenization, Studies in Mathematics and its Applications, vol. 26. Elsevier, Amsterdam (1992)

48. Ostoja-Starzewski, M.: Microstructural randomness and scaling in mechanics of materials. Chapman and Hall/CRC Press, Boca Raton (2007)

49. Sanchez-Palencia, E.: Non-homogeneous Media and Vibration Theory. Lecture Notes in Physics, vol. 127. Springer, Berlin (2000) 
50. Alibert, J.J., Della Corte, A.: Second-gradient continua as homogenized limit of pantographic microstructured plates: a rigorous proof. Zeitschrift für angewandte Mathematik und Physik 66(5), 2855-2870 (2015)

51. Alibert, J.J., Seppecher, P., dell'Isola, F.: Truss modular beams with deformation energy depending on higher displacement gradients. Math. Mech. Solids 8(1), 51-73 (2003)

52. Berlyand, L., Owhadi, H.: Flux norm approach to finite dimensional homogenization approximations with non-separated scales and high contrast. Arch. Ration. Mech. Anal. 198(2), 677$721(2010)$

53. Camar-Eddine, M., Seppecher, P.: Non-local interactions resulting from the homogenization of a linear diffusive medium. Comptes Rendus de l'Académie des Sciences-Series IMathematics 332(5), 485-490 (2001)

54. Camar-Eddine, M., Seppecher, P.: Closure of the set of diffusion functionals with respect to the mosco-convergence. Math. Models Methods Appl. Sci. 12(08), 1153-1176 (2002)

55. Carcaterra, A., dell'Isola, F., Esposito, R., Pulvirenti, M.: Macroscopic description of microscopically strongly inhomogenous systems: A mathematical basis for the synthesis of higher gradients metamaterials. Arch. Ration. Mech. Anal. 218(3), 1239-1262 (2015)

56. Challamel, N., Kocsis, A., Wang, C.: Higher-order gradient elasticity models applied to geometrically nonlinear discrete systems. Theor. Appl. Mech. 42(4), 223-248 (2015)

57. Cordero, N.M., Forest, S., Busso, E.P.: Second strain gradient elasticity of nano-objects. J. Mech. Phys. Solids 97, 92-124 (2016)

58. Javili, A., McBride, A., Mergheim, J., Steinmann, P., Schmidt, U.: Micro-to-macro transitions for continua with surface structure at the microscale. Int. J. Solids Struct. 50(16), 2561-2572 (2013)

59. Misra, A., Poorsolhjouy, P.: Identification of higher-order elastic constants for grain assemblies based upon granular micromechanics. Math. Mech. Complex Syst. 3(3), 285-308 (2015)

60. Pideri, C., Seppecher, P.: A second gradient material resulting from the homogenization of an heterogeneous linear elastic medium. Continuum Mech. Thermodynam. 9(5), 241-257 (1997)

61. Trinh, D.K., Janicke, R., Auffray, N., Diebels, S., Forest, S.: Evaluation of generalized continuum substitution models for heterogeneous materials. Int. J. Multiscale Computat. Eng. 10(6) (2012)

62. Naumenko, K., Eremeyev, V.A.: A layer-wise theory for laminated glass and photovoltaic panels. Comp. Struct. 112, 283-291 (2014)

63. Naumenko, K., Eremeyev, V.A.: A layer-wise theory of shallow shells with thin soft core for laminated glass and photovoltaic applications. Comp. Struct. 178, 434-446 (2017)

64. Besdo, D.: Towards a Cosserat-theory describing motion of an originally rectangular structure of blocks. Arch. Appl. Mech. 80(1), 25-45 (2010)

65. Bigoni, D., Drugan, W.J.: Analytical derivation of Cosserat moduli via homogenization of heterogeneous elastic materials. Trans. ASME J. Appl. Mech. 74(4), 741-753 (2007)

66. Dos Reis, F., Ganghoffer, J.: Construction of micropolar continua from the asymptotic homogenization of beam lattices. Comput. Struct. 112, 354-363 (2012)

67. Ehlers, W., Ramm, E., Diebels, S., d'Addetta, G.D.A.: From particle ensembles to Cosserat continua: Homogenization of contact forces towards stresses and couple stresses. Int. J. Solids Struct. 40(24), 6681-6702 (2003)

68. Goda, I., Ganghoffer, J.F.: Identification of couple-stress moduli of vertebral trabecular bone based on the 3d internal architectures. J. Mech. Behav. Biomed. Mater. 51, 99-118 (2015)

69. Goda, I., Assidi, M., Belouettar, S., Ganghoffer, J.: A micropolar anisotropic constitutive model of cancellous bone from discrete homogenization. J. Mech. Behav. Biomed. Mater. 16, 87-108 (2012)

70. Larsson, R., Diebels, S.: A second-order homogenization procedure for multi-scale analysis based on micropolar kinematics. Int. J. Numer. Methods Eng. 69(12), 2485-2512 (2007)

71. Reda, H., Rahali, Y., Ganghoffer, J., Lakiss, H.: Wave propagation in 3D viscoelastic auxetic and textile materials by homogenized continuum micropolar models. Comp. Struct. 141, 328 345 (2016) 
72. Trovalusci, P., De Bellis, M.L., Ostoja-Starzewski, M., Murrali, A.: Particulate random composites homogenized as micropolar materials. Meccanica 49(11), 2719-2727 (2014)

73. Trovalusci, P., Ostoja-Starzewski, M., De Bellis, M.L., Murrali, A.: Scale-dependent homogenization of random composites as micropolar continua. Eur. J. Mech. A/Solids 49, 396-407 (2015)

74. Trovalusci, P., De Bellis, M.L., Masiani, R.: A multiscale description of particle composites: From lattice microstructures to micropolar continua. Comp. Part B Eng. 128, 164-173 (2017)

75. Gurtin, M.E., Murdoch, A.I.: A continuum theory of elastic material surfaces. Arch. Ration. Mech. Anal. 57(4), 291-323 (1975)

76. Gurtin, M.E., Murdoch, A.I.: Surface stress in solids. Int. J. Sol. Struct. 14(6), 431-440 (1978)

77. Steigmann, D.J., Ogden, R.W.: Plane deformations of elastic solids with intrinsic boundary elasticity. Proc. Royal Soc. A 453(1959), 853-877 (1997)

78. Steigmann, D.J., Ogden, R.W.: Elastic surface-substrate interactions. Proc. Royal Soc. A 455(1982), 437-474 (1999)

79. Eremeyev, V.A.: On effective properties of materials at the nano-and microscales considering surface effects. Acta Mech. 227(1), 29-42 (2016)

80. Esposito, R., Pulvirenti, M.: From particles to fluids. Handbook of mathematical fluid dynamics 3, 1-82 (2004)

81. Pulvirenti, M.: Kinetic limits for stochastic particle systems. Lecture Notes in Mathematics pp. 96-126 (1996)

82. Saint-Raymond, L.: Hydrodynamic limits of the Boltzmann equation. 1971, Springer (2009)

83. Caprino, S., Esposito, R., Marra, R., Pulvirenti, M.: Hydrodynamic limits of the Vlasov equation. Commun. Partial Differ. Equat. 18(5-6), 805-820 (1993)

84. Carinci, G., De Masi, A., Giardinà, C., Presutti, E.: Hydrodynamic limit in a particle system with topological interactions. Arab. J. Math. 3(4), 381-417 (2014)

85. Carinci, G., De Masi, A., Giardinà, C., Presutti, E.: Super-hydrodynamic limit in interacting particle systems. J. Stat. Phys. 155(5), 867-887 (2014)

86. De Masi, A., Olla, S.: Quasi-static hydrodynamic limits. J. Stat. Phys. 161(5), 1037-1058 (2015)

87. De Masi, A., Merola, I., Presutti, E., Vignaud, Y.: Coexistence of ordered and disordered phases in Potts models in the continuum. J. Stat. Phys. 134(2), 243-306 (2009)

88. De Masi, A., Luckhaus, S., Presutti, E.: Two scales hydrodynamic limit for a model of malignant tumor cells. Annales de l'Institut Henri Poincare (B) Probability and Statistics 43(3):257-297 (2007)

89. De Masi, A., Galves, A., Löcherbach, E., Presutti, E.: Hydrodynamic limit for interacting neurons. J. Stat. Phys. 158(4), 866-902 (2015)

90. Papanicolaou, G.C., Varadhan, S.S.: Boundary value problems with rapidly oscillating random coefficients. Seria Colloq Math Society Janos Bolyai 1, 835-873 (1979)

91. Nadler, B., Papadopoulos, P., Steigmann, D.J.: Multiscale constitutive modeling and numerical simulation of fabric material. Int. J. Solids Struct. 43(2), 206-221 (2006)

92. Steigmann, D.J.: Two-dimensional models for the combined bending and stretching of plates and shells based on three-dimensional linear elasticity. Int. J. Eng. Sci. 46(7), 654-676 (2008)

93. Steigmann, D.J., dell'Isola, F.: Mechanical response of fabric sheets to three-dimensional bending, twisting, and stretching. Acta Mechanica Sinica 31(3), 373-382 (2015)

94. Giorgio, I.: Numerical identification procedure between a micro-Cauchy model and a macrosecond gradient model for planar pantographic structures. Zeitschrift für angewandte Mathematik und Physik 67(4), 95 (2016)

95. Saeb S, Steinmann P, Javili A (2016) Aspects of computational homogenization at finite deformations: A unifying review from reuss' to voigt's bound. Appl. Mech. Rev. 68(5):050,801

96. Bevill, G., Eswaran, S.K., Gupta, A., Papadopoulos, P., Keaveny, T.M.: Influence of bone volume fraction and architecture on computed large-deformation failure mechanisms in human trabecular bone. Bone 39(6), 1218-1225 (2006)

97. Ganghoffer, J.F.: Spatial and material stress tensors in continuum mechanics of growing solid bodies. Math. Mech. Comp. Syst. 3(4), 341-363 (2016) 
98. Berdichevsky, V.: Variational Principles of Continuum Mechanics: I. Fundamentals, Springer, Heidelberg (2009)

99. Hamilton, W.R.: On a general method in dynamics; by which the study of the motions of all free systems of attracting or repelling points is reduced to the search and differentiation of one central relation, or characteristic function. Philos. Trans. Royal Soc. London 124, 247-308 (1834)

100. Stigler, S.M.: Stigler's law of eponymy. Transactions of the New York Academy of Sciences 39 (1 Series II):147-157 (1980)

101. Benvenuto, E.: La scienza delle costruzioni e il suo sviluppo storico. Sansoni, Firenze (1981)

102. dell'Isola, F., Giorgio, I., Pawlikowski, M., Rizzi, N.: Large deformations of planar extensible beams and pantographic lattices: Heuristic homogenisation, experimental and numerical examples of equilibrium. Proc. Royal Soc. London Ser A 472(2185): 20150, 790 (2016)

103. Turco, E., Rizzi, N.L.: Pantographic structures presenting statistically distributed defects: numerical investigations of the effects on deformation fields. Mech. Res. Commun. 77, 65-69 (2016)

104. Turco, E., Barcz, K., Pawlikowski, M., Rizzi, N.L.: Non-standard coupled extensional and bending bias tests for planar pantographic lattices. part i: numerical simulations. Zeitschrift für angewandte Mathematik und Physik 67(5), 122 (2016)

105. Turco, E., Barcz, K., Pawlikowski, M., Rizzi, N.L.: Non-standard coupled extensional and bending bias tests for planar pantographic lattices. part i: numerical simulations. Zeitschrift für angewandte Mathematik und Physik 67(5), 122 (2016)

106. Turco, E., dell'Isola, F., Rizzi, N.L., Grygoruk, R., Müller, W.H., Liebold, C.: Fiber rupture in sheared planar pantographic sheets: Numerical and experimental evidence. Mech. Res. Commun. 76, 86-90 (2016)

107. Turco, E., Golaszewski, M., Cazzani, A., Rizzi, N.L.: Large deformations induced in planar pantographic sheets by loads applied on fibers: experimental validation of a discrete lagrangian model. Mech. Res. Commun. 76, 51-56 (2016)

108. Turco, E., Golaszewski, M., Giorgio, I., D’Annibale, F.: Pantographic lattices with nonorthogonal fibres: Experiments and their numerical simulations. Comp. Part B Eng. 118, 1-14 (2017)

109. Born, M., Huang, K.: Dynamical Theory of Crystal Lattices. Clarendon Press, Oxford, International series of monographs on physics (1954)

110. Ostoja-Starzewski, M.: Lattice models in micromechanics. Appl. Mech. Rev. 55(1), 35-60 (2002)

111. Porubov, A.: Modeling of strongly nonlinear effects in diatomic lattices. Arch. Appl. Mech. 84(9-11), 1533-1538 (2014)

112. Porubov, A., Andrianov, I.: Nonlinear waves in diatomic crystals. Wave Motion 50(7), 11531160 (2013)

113. Porubov, A., Antonov, I., Fradkov, A., Andrievsky, B.: Control of localized non-linear strain waves in complex crystalline lattices. Int. J. Non-Lin. Mech. 86, 174-184 (2016)

114. Porubov, A.V., Aero, E.L., Maugin, G.A.: Two approaches to study essentially nonlinear and dispersive properties of the internal structure of materials. Phys. Rev. E 79(4):046,608 (2009)

115. Russo, L.: The forgotten revolution: how science was born in $300 \mathrm{BC}$ and why it had to be reborn. Springer Science \& Business Media (2013)

116. Piola, D.G.: Sulla teorica dei cannocchiali. Memoria inserita nelle Effemeridi. Dall'Imp, Regia Stamperia, Milano (1821)

117. Piola, D.G.: Veglie di un filosofo. In: Soliani, E. (ed.) Memorie di religione, di morale e di letteratura. Tipografi Reale, Modena (1823)

118. dell'Isola, F., Maier, G., Perego, U., Andreaus, U., Esposito, R., Forest, S.: (eds) The complete works of Gabrio Piola: vol. I. Springer (2014)

119. Boutin, C., dell'Isola, F., Giorgio, I., Placidi, L.: Linear pantographic sheets: asymptotic micro-macro models identification. Math. Mech. Complex Syst. 5(2), 127-162 (2017)

120. Gatouillat, S., Bareggi, A., Vidal-Sallé, E., Boisse, P.: Meso modelling for composite preform shaping-simulation of the loss of cohesion of the woven fibre network. Comp. Part A Appl. Sci. Manufact. 54, 135-144 (2013) 
121. Harrison, P.: Modelling the forming mechanics of engineering fabrics using a mutually constrained pantographic beam and membrane mesh. Comp. Part A Appl. Sci. Manufact. 81, 145-157 (2016)

122. Harrison, P., Alvarez, M.F., Anderson, D.: Towards comprehensive characterisation and modelling of the forming and wrinkling mechanics of engineering fabrics. Int. J. Solids Struct. (2017)

123. Placidi, L., Barchiesi, E., Turco, E., Rizzi, N.L.: A review on $2 \mathrm{~d}$ models for the description of pantographic fabrics. Zeitschrift für angewandte Mathematik und Physik 67(5), 121 (2016)

124. Abali, B.E., Müller, W.H., Georgievskii, D.V.: A discrete-mechanical approach for computation of three-dimensional flows. ZAMM 93(12), 868-881 (2013)

125. Battista, A., Cardillo, C., Del Vescovo, D., Rizzi, N.L., Turco, E.: Frequency shifts induced by large deformations in planar pantographic continua. Nanomech. Sci.Technol. Int. J. 6(2), (2015)

126. Del Vescovo, D., Giorgio, I.: Dynamic problems for metamaterials: review of existing models and ideas for further research. Int. J. Eng. Sci. 80, 153-172 (2014)

127. Lejeune, E., Javili, A., Linder, C.: An algorithmic approach to multi-layer wrinkling. Extreme Mech. Lett. 7, 10-17 (2016)

128. Placidi, L., Greco, L., Bucci, S., Turco, E., Rizzi, N.L.: A second gradient formulation for a $2 \mathrm{~d}$ fabric sheet with inextensible fibres. Zeitschrift für angewandte Mathematik und Physik 67(5), 114 (2016)

129. Pideri C, Seppecher P (2006) Asymptotics of a non-planar rod in non-linear elasticity. Asymp. Anal. 48(1, 2):33-54

130. Placidi, L., Andreaus, U., Della Corte, A., Lekszycki, T.: Gedanken experiments for the determination of two-dimensional linear second gradient elasticity coefficients. Zeitschrift für angewandte Mathematik und Physik 66(6), 3699-3725 (2015)

131. Placidi, L., Andreaus, U., Giorgio, I.: Identification of two-dimensional pantographic structure via a linear d4 orthotropic second gradient elastic model. J. Eng. Math. 103(1), 1-21 (2017)

132. Andreaus, U., Giorgio, I., Lekszycki, T.: A 2-d continuum model of a mixture of bone tissue and bio-resorbable material for simulating mass density redistribution under load slowly variable in time. ZAMM J. Appl. Mathematics and Mechanics/Zeitschrift für Angewandte Mathematik und Mechanik 94(12), 978-1000 (2014)

133. Giorgio, I., Andreaus, U., Scerrato, D., dell'Isola, F.: A visco-poroelastic model of functional adaptation in bones reconstructed with bio-resorbable materials. Biomech. Model. Mechanobiol. 15(5), 1325-1343 (2016)

134. Giorgio, I., Andreaus, U., Lekszycki, T., Corte, A.D.: The influence of different geometries of matrix/scaffold on the remodeling process of a bone and bioresorbable material mixture with voids. Math. Mech. Solids 22(5), 969-987 (2017)

135. Giorgio, I., Andreaus, U., Scerrato, D., Braidotti, P.: Modeling of a non-local stimulus for bone remodeling process under cyclic load: Application to a dental implant using a bioresorbable porous material. Math. Mech. Solids 22(9), 1790-1805 (2017) 